\title{
DEFORMATION MODES FOR ASSEMBLIES OF FRICTIONLESS POLYDISPERSE SPHERES
}

\author{
N. Kumar, O. I. Imole, V. Magnanimo and S. Luding \\ Multi-Scale Mechanics, TS, CTW, University of Twente \\ P.O. Box 217, 7500 AE Enschede, Netherlands \\ Email: n.kumar@utwente.nl, Tel.: +31 534893061
}

Keywords: DEM; uniaxial deformation; pure shear deformation; deviatoric stress and strain

\begin{abstract}
The challenge of dealing with cohesive powders during storage, handling and transport are widely known in the process and pharmaceutical industries. Simulations with the discrete element method (DEM) provide further insight into the local microstructure of bulk materials. In this work, the DEM approach is presented to investigate the flow behavior of granular systems subjected to different modes of deformations. When uniaxial compression is applied of frictionless, polydisperse spheres above jamming (transition from fluid-like state to solid-like state), the evolution of coordination number (average number of contacts per particle) and pressure as functions of the volume fraction are, astonishingly, identical to results obtained for purely isotropic compression. Analytical predictions for the evolution of pressure and coordination number under isotropic strain can thus be separated from different deformation modes, as applied in this study. After two different modes of volume-conserving deviatoric shear, the results still compare quite well with results for purely isotropic compression. The difference between the two deviatoric modes and uniaxial deformation is examined with respect to the anisotropic stress response as a function of deviatoric strain.
\end{abstract}

\section{Introduction}

Dense granular materials are complex systems, which show unique material properties, different from classical fluids or solids. This involves phenomena like dilatancy, shear band formation, history dependence, jamming, and yield stress, among others. A full understanding of the flow and deformation behavior, especially for fine, cohesive powders, still remains a challenging problem.

It has been shown [1] that isotropic and deviatoric deformation modes are pure modes, while the uniaxial deformation test derives from the superposition of an isotropic and a deviatoric test. In this work, various deformations paths for aggregates of polydisperse packings of non-frictional particles are modeled using the DEM simulation approach. The evolution of coordination number, deviatoric stress, and pressure (isotropic stress) are reported as functions of isotropic and deviatoric strain for different deformation modes and material parameters in Ref. [8].

\section{Simulation procedure}

The Discrete Element Method (DEM) [3] can be used to perform simulations in a triaxial box. One great advantage of the triaxial box is the possibility of realizing different deformation modes with a single test experiment (element test) applying direct control of stress or strain [1]. For the sake of simplicity, the contact model used in this work is the linear visco-elastic normal force

$$
f^{n}=k \delta+\gamma \dot{\delta}
$$

where $k$ is the spring stiffness, $\gamma$ the contact viscosity parameter, $\delta$ the overlap between particles and $\dot{\delta}$ the relative velocity in the normal direction. In order to reduce dynamical effects and shorten relaxation times, an artificial background viscous dissipation force $f_{b}=-\gamma_{b} v_{i}$ proportional to the moving particle $i$ with velocity $v_{i}$ is added, resembling the damping due to a background medium. 


\subsection{Simulation parameters}

Simulation parameters are the system size $N=9261\left(=21^{3}\right)$ particles, density $\rho=2000\left[\mathrm{~kg} / \mathrm{m}^{3}\right]$, elastic stiffness $k_{n}=10^{5}\left[\mathrm{~kg} / \mathrm{s}^{2}\right]$, particle damping coefficient $\gamma=1[\mathrm{~kg} / \mathrm{s}]$, and background dissipation $\gamma_{b}=0.1[\mathrm{~kg} / \mathrm{s}]$. Ref. [4] provides a description of these artificial units and how they could be rescaled to fit values obtained from experiments. It should also be noted that the system has average particles radius $\langle r\rangle=1$ [mm], with polydispersity quantified by the width $w=r_{\max } / r_{\min }=3$ of a uniform distribution.

\subsection{Initial configuration}

The boundary conditions of the triaxial cell are periodic walls and are strain controlled. The initial configuration is such that particles were randomly generated in a 3D box and isotropically compressed to a target volume fraction $v_{0}=0.673$ above the volume fraction. From there, the isotropic compression stage is continued as the conditioning or preparation stage for various volume fractions, before the initiation of the uniaxial and deviatoric tests, as schematically shown in Fig. 1.

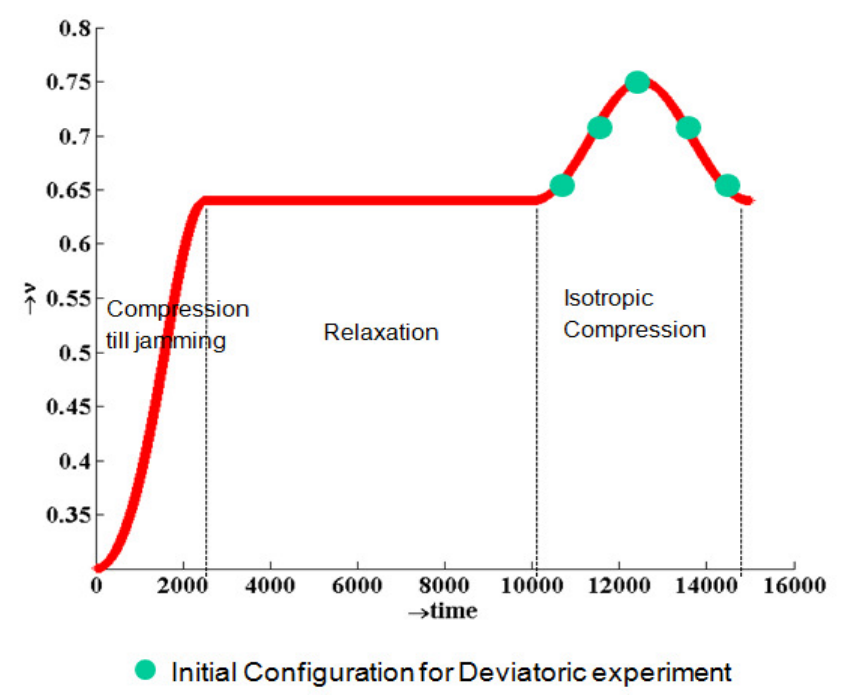

Fig. 1: Volume fraction vs time during the isotropic preparation, relaxation and further isotropic compression and decompression stages. Cyan color represents various initial configurations for the deviatoric deformation.

\section{(a) Uniaxial deformation}

Uniaxial compression is subsequently initiated at the end of the isotropic stage, after allowing for sufficient relaxation of the isotropic system, with diagonal strain matrix elements $\varepsilon_{U}(0,0,1)$ where positive values mean compression. The volume fraction increases with time during uniaxial compression to a maximum of $v_{\max }=0.82$ and is followed by decompression back to the original $v_{0}$.

\section{(b) Deviatoric deformation}

After the isotropic preparation deviatoric (volume conserving) tests are performed. We choose two different ways of deforming the system. One case is with (diagonal) strain tensor (elements), $\varepsilon_{\mathrm{D} 2}(-1,0,1)$, where only 2 sides are moving. $\varepsilon_{\mathrm{D} 2}$ is the strain-magnitude and the diagonal elements set the type and direction of triaxial strain. In this case, one wall moves outside as much as the other wall moves inside, with the third wall fixed. The second deviatoric deformation mode is described by the strain matrix $\varepsilon_{\mathrm{D} 3}(-1 / 2,-1 / 2,1)$. All the three walls move with one wall twice as much as the other two. Several other configurations can be chosen to perform deviatoric deformation, but we are mainly interested in these two fundamental modes. We perform slow deformations to allow the system to relax and hence be in the quasi-static regime.

\subsection{Evolution of Coordination Number}

Jamming occurs at the isostatic point $[2,5]$. The contacts of dynamic rattlers (that do not contribute to the force network) are transient because the repulsive contact forces will push them away from the 
mechanically stable backbone [2]. We define frictionless particles with less than 4 contacts as rattlers. This leads to the following definition:

$N_{4}:=N_{C \geq 4}:$ Number of particles with at least 4 contacts,

$M_{4}:=M_{C \geq 4}$ : Total number of contacts of $N_{4}$ particles

$C^{*}:=\frac{M_{4}}{N_{4}}: \quad$ Corrected Coordination number

The plot with variation of $C^{*}$ can be seen in Fig. 2. We observe that $C^{*}$ follows a power law with volume fraction

$$
\mathrm{C}^{*}(v):=\mathrm{C}_{0}+\mathrm{C}_{1}\left(\frac{v}{v_{\mathrm{c}}}-1\right)^{\alpha}
$$

where $\mathrm{C}_{0}=6, \mathrm{C}_{1} \approx 8.164$ and $\alpha \approx 0.57$ are the fitted parameters and $v_{\mathrm{c}} \approx 0.665$.

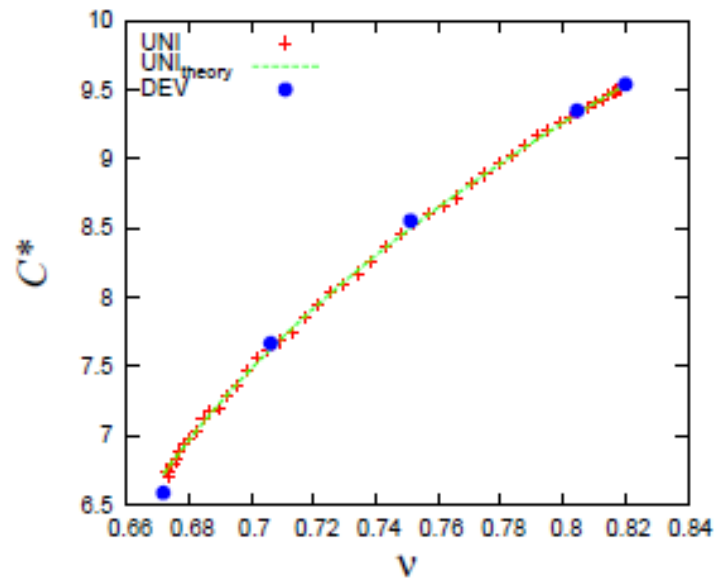

Fig. 2: Comparison of $C^{*}$ with the numerical data (red) and the proposed fit (green), Eq. (2). The blue dots represent the asymptotic values of $C^{*}$ for various different volume fractions after large strain deviatoric deformation.

One interesting observation is that the coordination number, shown in Fig. 2, also is described by isotropic and uniaxial data - at the end of deviatoric simulations with different initial volume fractions (since volume is conserved under pure shear deformation). This suggests that the coordination number evolution equation proposed in Eq. (2), is independent of the deviatoric part of deformation (when particles are frictionless).

\subsection{Evolution of pressure}

The non-dimensional pressure [2] can be defined as:

$$
p=\frac{2\langle r\rangle}{3 k} \operatorname{tr}(\sigma)
$$

where $\operatorname{tr}(\sigma)$ is the trace of the averaged stress tensor and $\langle r\rangle$ is the mean radius of the spheres. The non-dimensional pressure - strain relation is [2]:

$$
p=p_{0} \frac{v C}{v_{c}}\left(-\varepsilon_{v}\right)\left[1-\gamma_{p}\left(-\varepsilon_{v}\right)\right]
$$

where $p_{0}, \gamma_{p}$ are the fit parameters, $C=C^{*}\left(1-\phi_{r}\right)$ is the coordination number $\left(\phi_{r}\right.$ is the rattler fraction [2]) and $\varepsilon_{v}$ is the true logarithmic volume change integral of $\epsilon_{v}=\epsilon_{i i}$ of the system. Fig. 3 shows the total pressure as a function of the volume fraction for the Uniaxial, Isotropic and Deviatoric deformation modes. When the non-dimensional pressure is plotted versus the volume fraction, the deviatoric data (blue dots) also almost collapse with the two other deformation modes with slight deviations possibly due to dilatancy or small dynamic effects [8]. 


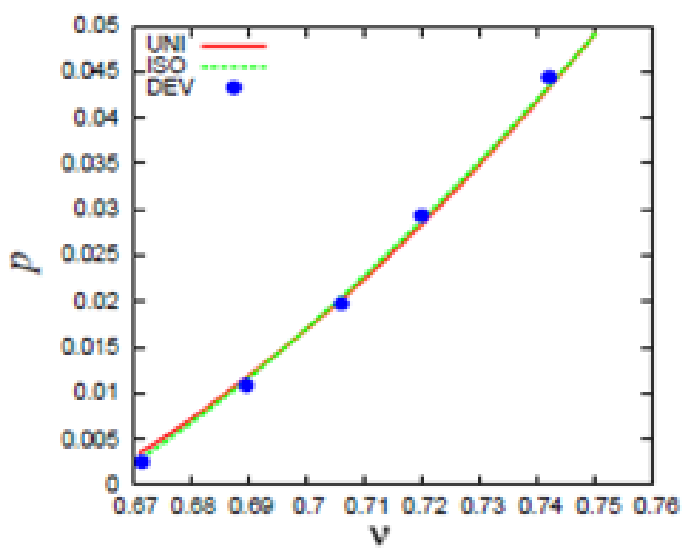

Fig. 3: Non-dimensional pressure plotted against volume fraction for the UNIaxial, ISOtropic and DEViatoric deformation modes.

For all cases (isotropic, uniaxial and deviatoric), the coefficients $p_{0} \approx 0.039, \gamma_{p} \approx 0.011$ and $v_{c} \approx 0.665$ fit our data well with errors of less than one percent, while the deviatoric data are off by $1-2 \%$ only.

\subsection{Deviatoric stress}

The average isotropic stress (pressure) is defined here as:

$$
p=\frac{\sigma_{x x}+\sigma_{y y}+\sigma_{z z}}{3}=\frac{1}{3} \operatorname{tr}(\sigma)
$$

where $\sigma_{x x}, \sigma_{y y}$ and $\sigma_{z z}$ are the diagonal elements of the stress tensor. Several definitions are available in literature [6] to define the deviatoric stress. We propose the most objective form to calculate the shear stresses [7] for the 3D box that accounts for triaxial deformation:

$$
\sigma_{D E V}=\sqrt{\frac{\left(\sigma_{x x}-\sigma_{y y}\right)^{2}+\left(\sigma_{y y}-\sigma_{z z}\right)^{2}+\left(\sigma_{z z}-\sigma_{x x}\right)^{2}}{2}}
$$

We can also define the deviatoric strain in a similar way as:

$$
\varepsilon_{D E V}=\sqrt{\frac{\left(\varepsilon_{x x}-\varepsilon_{y y}\right)^{2}+\left(\varepsilon_{y y}-\varepsilon_{z z}\right)^{2}+\left(\varepsilon_{z z}-\varepsilon_{x x}\right)^{2}}{2}}
$$

where $\varepsilon_{x x}, \varepsilon_{y y}$ and $\varepsilon_{z z}$ are the diagonal elements of the strain matrix. We use the factor 2 in the denominator of Eqs. (6) and (7) following the definition of the second stress invariant, even though different factors have been proposed in literature [6], which result in a change in the maximum anisotropy values obtained. The deviatoric stress $\sigma_{D E V}$ quanitfies the (stress) anisotropy between the compression/de-compression and inactive directions. The response for our two different deviatoric simulations is shown in Fig. 4(a) and Fig. 4(b). The deviatoric stress has been normalized with the average isotropic stress $p$ from Eq. (5). Results for different volume fractions between $v=0.666$ to $v=0.82$ are shown.

For the deviatoric deformation modes D2 and D3, the stresses grow with applied deviatoric strain until an asymptotic behaviour (maximum stress anisotropy) is reached. This is in agreement with the constitutive model for volume conserving deformations in the biaxial box proposed in [1,7] and studied elsewhere [8]. Interestingly, the evolution of the stress-ratios is very similar for the different deformation modes. Also, the slope of the normalized deviatoric stress function against deviatoric strain reduces as the volume fraction is increased, unlike the classical shear modulus $G$, which increases with volume fraction [8], as consistent with findings from macroscopic experiments with shear testers [9]. 

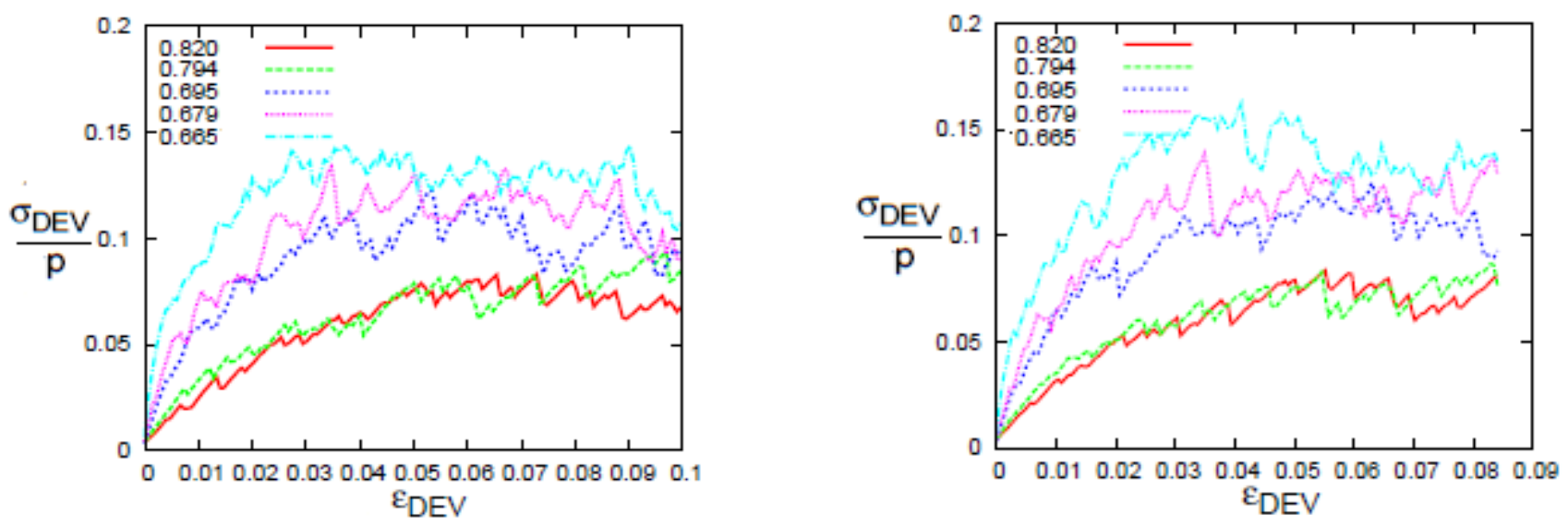

Fig. 4(a): Evolution of deviatoric stress as a function of deviatoric strain for deviatoric mode D2 with strain matrix $\varepsilon_{D 2}(-1,0,1)$. Fig. 4(b): Plot of deviatoric stress as a function of deviatoric strain for deviatoric mode D3 with strain matrix $\varepsilon_{D 2}(-1 / 2,-1 / 2,1)$.

\section{Conclusions}

We have presented simulation results from strain controlled uniaxial compression and deviatoric (pure shear) deformation of frictionless polydisperse spheres. An important result in this study is the agreement obtained between the data for all deformations modes with the analytical predictions for purely volumetric strain [2]. When the non-dimensional pressure is plotted against the volume fraction, the data collapse on a unique law, irrespective of the applied deformation mode, purely isotropic, uniaxial and deviatoric (D2 \& D3). This suggests an advantage of the 'cheaper' uniaxial (and deviatoric) deformation modes over the hard to realize isotropic deformation. Three walls have to be moved simultaneously in the isotropic case, while less movement is required in the other modes for the same amount of deformation. Symmetry in the two non-mobile directions for UNI and D3 modes is confirmed. For further work, more realistic contact models able to take into account friction and cohesion, need to be implemented.

\section{Acknowledgement}

Helpful discussions with A. Singh and F. Göncü are gratefully acknowledged. This work is financially supported by the research programme "PARDEM", under Marie Curie ITN, European Union support.

\section{References}

[1] Luding, S., and Perdahcioglu, S., "A local constitutive model with anisotropy for various homogeneous 2D Biaxial deformation modes", CIT 83(5), pp. 672-688, 2011.

[2] Göncü, F., Duran, O., and Luding, S., "Constitutive relations for the isotropic deformation of frictionless packings of polydisperse spheres”, C. R. Mecanique, 338, pp. 570-586, 2010.

[3] Cundall P.A., Strack, O.D.L., "A discrete numerical model for granular assemblies”. Geotechnique, 29, pp. 47-65, 1979.

[4] Luding, S., "Cohesive, frictional powders: contact models for tension," Granular Matter, 10, pp. 235-246, 2008.

[5] O’Hern, C. S., Silbert L. E., Liu A. J., and Nagel S. R., "Jamming at zero temperature and zero applied stress: The epitome of disorder," Phys. Rev. E, 68, Part 1, 2003.

[6] Magnanimo, V. and Luding, S., "A local constitutive model with anisotropy for ratcheting under 2D biaxial isobaric deformation “, Granular Matter, 13, No. 3, pp. 225-232.

[7] Imole, O. I., Kumar, N. and Luding, S., "Deformation modes of packings of frictionless polydisperse spheres" in press, PSA2011 proceedings, Edinburgh, 5-7 Sept., 2011

[8] Imole, O. I., Kumar, N. and Luding, S., "Different deformation modes of 3 dimensional packings of frictionless polydisperse spheres" paper in preparation.

[9] Schwedes, J. and Schulze, D., "Measurement of flow properties of bulk solids", Powder Technology, 61, pp. 59-68, 1990 